\title{
Reconstruction of solar irradiance since 1610: Implications for climate change
}

\author{
Judith Lean ${ }^{1}$, Juerg Beer ${ }^{2}$ and Raymond Bradley ${ }^{3}$
}

\begin{abstract}
Solar total and ultraviolet (UV) irradiances are reconstructed annually from 1610 to the present. This epoch includes the Maunder Minimum of anomalously low solar activity (circa 1645-1715) and the subsequent increase to the high levels of the present Modern Maximum. In this reconstruction, the Schwabe (11-year) irradiance cycle and a longer term variability component are determined separately, based on contemporary solar and stellar monitoring. The correlation of reconstructed solar irradiance and Northern Hemisphere (NH) surface temperature is 0.86 in the pre-industrial period from 1610 to 1800 , implying a predominant solar influence. Extending this correlation to the present suggests that solar forcing may have contributed about half of the observed $0.55^{\circ} \mathrm{C}$ surface warming since 1860 and one third of the warming since 1970 .
\end{abstract}

\section{Introduction}

From 1860 to 1990 , the global mean annual surface temperature increased $0.55^{\circ} \mathrm{C}$ [Parker et al., 1994]. At the same time, the concentration of industrially produced $\mathrm{CO}_{2}$ gas in the Earth's atmosphere increased from 280 to 353 ppmv, leading to the hypothesis that the warmer temperatures signify the climate system's response to anthropogenic influences [IPCC, 1992]. However, statistical analyses of the climate record since 1860 reveal significant interannual and interdecadal variability [Allen and Smith, 1994; Mann and Park, 1994], suggesting that the cause of the warming is more complex than the influence of increasing greenhouse gases alone. Furthermore, the surface temperature increase of the past 130 years appears to be part of a longer term warming that commenced in the seventeenth century [Bradley and Jones, 1993], prior to the industrial epoch. Paleoclimate records obtained from ice-cores, tree-rings, pollen, corals and glacial events likewise attest to the variability of the pre-industrial climate.

Natural influences of solar variability and volcanic eruptions potentially impact the Earth's surface temperature [Rind and Overpeck, 1993] but these effects have yet to be specified with the certainty needed to extract any residual anthropogenic signal. Like the greenhouse gas concentrations, the overall activity level of the Sun has risen steadily during the past 130 years. Surface temperatures correlate well with solar activity over this period

\footnotetext{
'E.O. Hulburt Center for Space Research, Naval Research Laboratory, Washington, DC

${ }^{2}$ Swiss Federal Institute for Environmental Science and Technology, Dübendorf, Switzerland

${ }^{3}$ Department of Geosciences, University of Massachusetts, Amherst, MA
}

Copyright 1995 by the American Geophysical Union.

Paper number 95GL03093

0094-8534/95/95GL-03093\$03.00
[Reid, 1991; Friis-Christensen and Lassen, 1991], with correlation coefficients as high (0.7) as the correlation between surface temperatures and greenhouse gas concentrations. However, attempts to account for the entire surface warming of the industrial epoch by solar radiative forcing alone have been refuted because simulations of this scenario with simple climateocean energy balance models require climate sensitivities beyond the expected conservative range of 0.3 to $1.0^{\circ} \mathrm{C} / \mathrm{Wm}^{-2}$, or solar irradiance variability larger than the $0.1 \%$ change observed over a recent Schwabe (11-year) cycle [Schlesinger and Ramankutty, 1992; Kelly and Wigley, 1992].

Analysis of the variability of ionized $\mathrm{Ca}$ emission, a magnetic activity surrogate in the Sun and stars, suggests that solar total irradiance during the seventeenth century Maunder Minimum [Eddy, 1976] may have been reduced by $0.24 \%$ relative to the present day mean [Lean et al., 1992], a larger change than the $0.1 \%$ 11-year cycle amplitude. As well, accompanying reductions in solar UV irradiance may have exceeded (by about a factor of two) the Schwabe cycle amplitudes of $3 \%$ at $250 \mathrm{~nm}$ and $7 \%$ at $200 \mathrm{~nm}$ [Lean et al., 1995].

With the goal of investigating solar forcing of climate change prior to and during the industrial epoch, we reconstruct annual solar total irradiance since 1610 by combining the Schwabe cycle with a longer term variability component, and compare this reconstruction with independent records of solar activity obtained from cosmogenic isotopes in tree-rings and ice-cores [Stuiver and Braziunas, 1993; Beer et al., 1994]. We then determine linear correlations between decadal means of our reconstructed irradiance and $\mathrm{NH}$ surface temperature anomalies [Bradley and Jones, 1993] in the pre-industrial period from 1610 to 1800 , and assess the extent to which solar induced surface temperatures estimated by the pre-industrial parameterization account for the post industrial warming.

We also reconstruct annual UV (200 to $300 \mathrm{~nm}$ ) irradiance since 1610 . This radiation contributes significantly to total irradiance variations [Lean, 1989] but is absorbed in the Earth's atmosphere. Variable UV irradiance may impact climate indirectly by modulating the ozone layer and hence the radiative and dynamical coupling of the stratosphere and the troposphere. Model studies have recently begun to investigate the possible climatic impacts of UV irradiance variations [Haigh, 1994; Rind and Balachandran, 1995].

\section{Parameterization of Present Day Solar Irradiance Variability}

Direct solar monitoring extends only over the past 15 years. To estimate solar irradiance outside this time base, extant irradiance time series are related to solar activity indices, such as the sunspot number, that extend back in time. This approach first requires proper quantification of irradiance variability sources in the contemporary solar monitoring database.

Solar irradiance varies during the Schwabe cycle because bright solar faculae and dark sunspots modulate the Sun's 

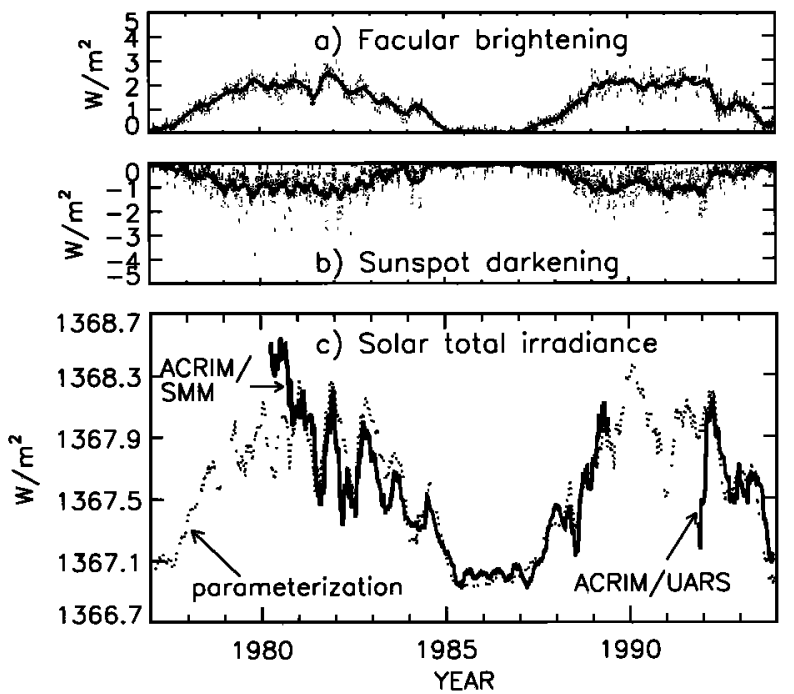

Figure 1. Shown are (a) bolometric facular brightening parameterized using $\mathrm{He} 1083 \mathrm{~nm}$ EW data, (b) sunspot darkening calculated from white light solar images, and (c) their net modulation of total irradiance during the Schwabe cycle, compared with measurements made by ACRIM on the SMM and UARS spacecraft, cross-calibrated using overlapping ERBS observations. Deviations of the SMM and UARS data from the reconstructed irradiances in 1980 and 1992, respectively, may reflect instrumental effects in the ACRIM data, since space-based radiometers are most susceptible to sensitivity changes during their first year of operation.

radiation. Both faculae and sunspots are magnetic phenomena that occur more frequently during times of high solar activity. At the visible wavelengths that dominate total solar radiative output, facular emission near solar activity maximum exceeds the corresponding sunspot deficit by a factor of 1.5 , causing a net total irradiance increase. Facular emission becomes increasingly larger than sunspot darkening at shorter UV wavelengths, which also vary in phase with solar activity.

Parameterizations of bolometric (i.e., spectrally integrated) facular brightening and sunspot darkening are shown in Figure 1. The sunspot darkening was calculated from information about sunspot areas and disk locations from white light solar images. Facular brightening was derived from observations by the Active Cavity Radiometer Irradiance Monitor (ACRIM I) on the Solar Maximum Mission (SMM) Spacecraft [Willson and Hudson, 1991] by correlating the measured irradiance corrected for sunspot darkening (called the residual irradiance) with the He $1083 \mathrm{~nm}$ equivalent width (EW) proxy. Figure 1 shows that the shape of the net modulation of total irradiance by sunspots and faculae compares well with the ACRIM I data, from which the parameterizations were deduced, and also with measurements by ACRIM II on the Upper Atmosphere Research Satellite (UARS), adjusted to the ACRIM I irradiance scale by using overlapping total irradiance observations from the Earth Radiation Budget Satellite (ERBS) [Lee III et al., 1995].

UV irradiance variations have a similar shape to the facular brightening in Figure 1 with a Schwabe cycle of roughly $1 \%$ amplitude in the integrated radiation from 200 to $300 \mathrm{~nm}$ [Lean et al., 1995]. This magnitude of variability is generally consistent with UV irradiance monitoring by the UARS, but rigorous validation is precluded as the long term precision of solar UV monitoring instruments is no better than $1 \%$.

\section{Reconstruction of Historical Solar Irradiance}

Our reconstruction of solar irradiance since 1610 is based on parameterizations of sunspot darkening and facular brightening analogous to those in the previous section, but using the sunspot record of solar activity that extends over almost four centuries. The reconstruction consists of two separate components; an 11year cycle plus a slowly varying background. The amplitude of each component is deduced separately from the database of solar and stellar (in the case of the long term component) monitoring. The temporal shape of each component is related separately to a newly reported group sunspot number record of solar activity. Group sunspot numbers, which are based on recent assessment of newly compiled historic sunspot observations and the identification of errors in Wolf's compilation, are similar to Wolf sunspot numbers after 1880 but are lower, by up to a factor of two, in the eighteenth century [Hoyt et al., 1994].

Shown in Figure 2 (thin line) is the solar irradiance reconstructed with the Schwabe cycle as the only cause of variability. After 1874 , total irradiance Schwabe cycles are reconstructed [following Foukal and Lean, 1990] using the sunspot darkening calculated from Greenwich Observatory white light solar images and a facular parameterization determined by correlating monthly mean sunspot numbers with ACRIM residual (i.e., sunspot corrected) irradiances. Prior to 1874 the reconstruction uses direct correlation of yearly mean total irradiance and group sunspot number. The 200-300 nm UV Schwabe cycle (Fig. 2b, thin line) is also derived from a linear transformation of the yearly mean group sunspot number such that the amplitude of the most recent cycle is about $1 \%$.

Long term changes in solar total irradiance reconstructed with the Schwabe cycle as the only cause of variability are of the order of $0.05 \%$. These changes are shown in Figure 2a to arise from increasing Schwabe cycle amplitudes since 1610 , a period including the Maunder Minimum in which the Schwabe sunspot cycle apparently ceased for a number of decades. There is evidence from Sunlike stars for weaker overall emission from
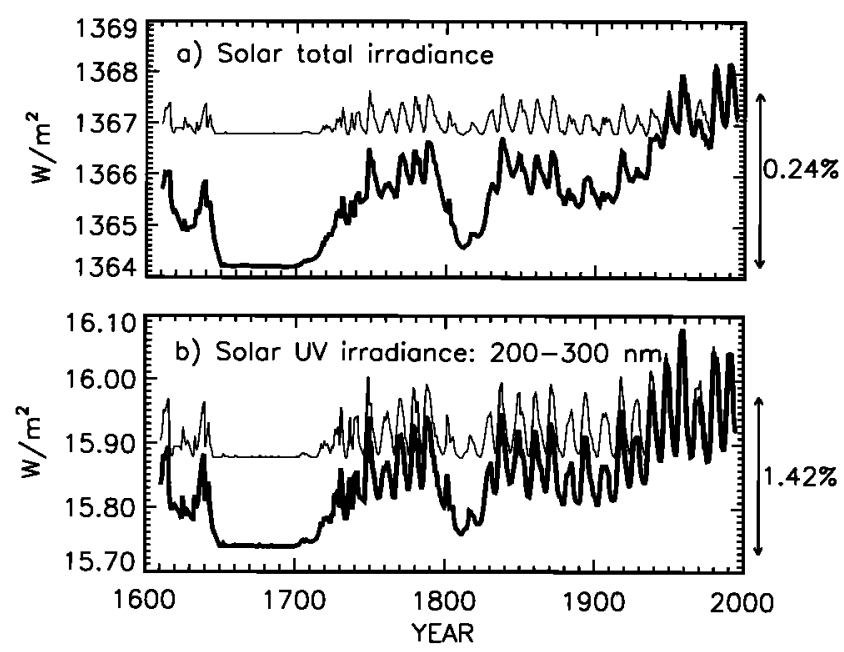

Figure 2. Reconstruction from 1610 to the present of (a) solar total irradiance and (b) UV irradiance in the band from 200 to $300 \mathrm{~nm}$. In each panel the thin line is the irradiance variability of the Schwabe cycle, and the thick line is the Schwabe cycle plus a longer term variability component needed to account for the amplitude of irradiance reductions estimated independently for the Maunder Minimum (1645-1715) from observations of Sunlike stars. 
noncycling stars in states analogous to the Maunder Minimum, relative to their emission levels in cycling states typified by the contemporary Sun [Baliunas and Jastrow, 1990]. Consistent with this, the Sun's present emission of ionized $\mathrm{Ca}$ (a surrogate for magnetic activity associated with bright facular regions on the Sun) is typical of the brightest one third of a sample of 13 Sunlike stars [White et al., 1992]. Using the relationship between solar Ca emission and ACRIM residual irradiances, Lean et al. [1992] estimated that a reduction in solar $\mathrm{Ca}$ emission to levels exhibited by noncycling Sunlike stars (i.e., the Maunder Minimum Sun) corresponds to a reduction of $0.24 \%$ in solar total irradiance. The proposed mechanism is depletion of the bright facular network that normally covers the Sun, even during times of Schwabe cycle minima, and an additional reduction in the emission from the non network regions to values presently seen in only the $15 \%$ darkest regions on the solar disk [White et al., 1992].

In order to replicate this quantitative deduction from Sunlike stars of the irradiance reduction during the seventeenth century, a second scenario shown in Figure 2 combines the Schwabe irradiance cycle with a longer term irradiance variability component. Following the demonstration by Foukal and Lean [1990] that facular brightening tracks the monthly mean sunspot number throughout the Schwabe cycle, we assume that on longer time scales the network facular emission likewise tracks the overall level of solar activity, and specify the shape of these changes by the average amplitude of the group sunspot number in each Schwabe cycle. This longer term component is scaled to cause an increase of $0.19 \%$ in solar total irradiance and $0.97 \%$ in UV $(200-300 \mathrm{~nm})$ irradiance from the Maunder Minimum to the present. Including the Schwabe cycle, overall variability from the Maunder Minimum to the present-day mean is thus constrained to agree with a solar total irradiance change of $0.24 \%$ [Lean et al., 1992] and a 200-300 nm UV irradiance change of $1.42 \%$ [Lean et al., 1995].

The total irradiance reconstruction is shown in Figure 3 to track independent records of solar activity levels inferred from ${ }^{14} \mathrm{C}$ and ${ }^{10} \mathrm{Be}$ cosmogenic isotopes [Stuiver and Braziunas, 1993; Beer et al., 1994]. However, this reconstruction differs noticeably (Figure 3) from that of Hoyt and Schatten [1993], which has a long term variability component based on the length (rather than amplitude) of the Schwabe cycle. These differences, which cannot be resolved without improved understanding of the solar origins of the variations, reflect the large uncertainties in

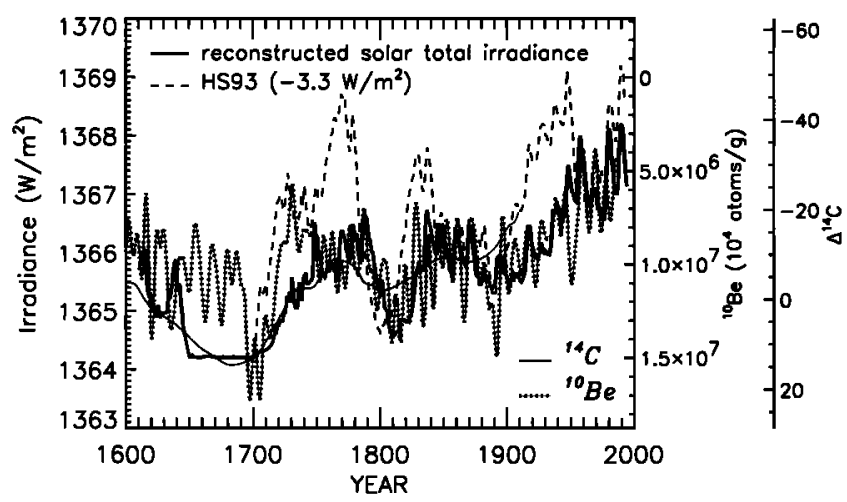

Figure 3. Tree-ring ${ }^{14} \mathrm{C}$ and ice-core ${ }^{10} \mathrm{Be}$ cosmogenic isotope records of solar variability are compared with reconstructed solar total irradiance and with the irradiance reconstruction of Hoyt and Schatten [1993] (HS93) which has a long term component based on Schwabe cycle length (rather than average amplitude).
Table 1. Correlation of decadal means of solar irradiance, $\mathrm{NH}$ dust veil index and $\mathrm{CO}_{2}$ concentrations with $\mathrm{NH}$ surface temperature anomalies, comprising the Bradley and Jones [1993] $\mathrm{NH}$ summer data from 1610 to 1850 , scaled to IPCC [1992] NH annual data, and the IPCC NH data since then.

\begin{tabular}{lcc}
\hline Climate Forcing & \multicolumn{2}{c}{ Correlation Coefficient } \\
& $1610-1800$ & $1800-2000$ \\
\hline Solar total irradiance & 0.86 & 0.75 \\
$\mathrm{NH}$ dust veil index & 0.005 & -0.55 \\
$\mathrm{CO}_{2}$ & 0.70 & 0.85 \\
\hline
\end{tabular}

reconstructing historical solar irradiances from a limited solar monitoring database, with only rudimentary knowledge of the pertinent physical processes.

\section{Climate Change Implications}

We investigate solar forcing of climate change since 1610 using decadal averages of our reconstructed solar irradiance and NH surface temperature anomalies [Bradley and Jones, 1993] scaled to match the IPCC [1992] NH data as shown in Figure 4. From 1610 to 1800 the correlation of surface temperature with solar irradiance is 0.86 but negligible with the $\mathrm{NH}$ dust veil index (Table 1), suggesting a predominant solar influence during this pre-industrial period. Extending the relationship between surface temperature anomalies $\Delta \mathrm{T}$ and solar total irradiance $\mathrm{S}(\Delta \mathrm{T}=$ $-200.44+0.1466 \times S)$ to the present yields a solar induced surface warming of $0.51^{\circ} \mathrm{C}$ since the Maunder Minimum, in surprisingly good agreement with the $\mathrm{NH}$ equilibrium surface temperature change of $0.49^{\circ} \mathrm{C}$ simulated by the Goddard Institute for Space Studies general circulation climate model (whose sensitivity is in the range 0.7 to $1^{\circ} \mathrm{C} / \mathrm{Wm}^{-2}$ ) for $\Delta S=0.25 \%$ [Rind and Overpeck, 1993]. Surface temperature cooling in the nineteenth century relative to the expected solar related warming may reflect extended volcanic activity during this period.

Since 1860 about half of the observed $0.55^{\circ} \mathrm{C}$ surface warming is attributable to direct solar forcing, according to our simple pre-industrial parameterization. But $0.36^{\circ} \mathrm{C}$ of this warming has occurred since 1970 , and solar forcing can account for only $0.11^{\circ} \mathrm{C}$ (less than a third) of this. Nor does our reconstruction

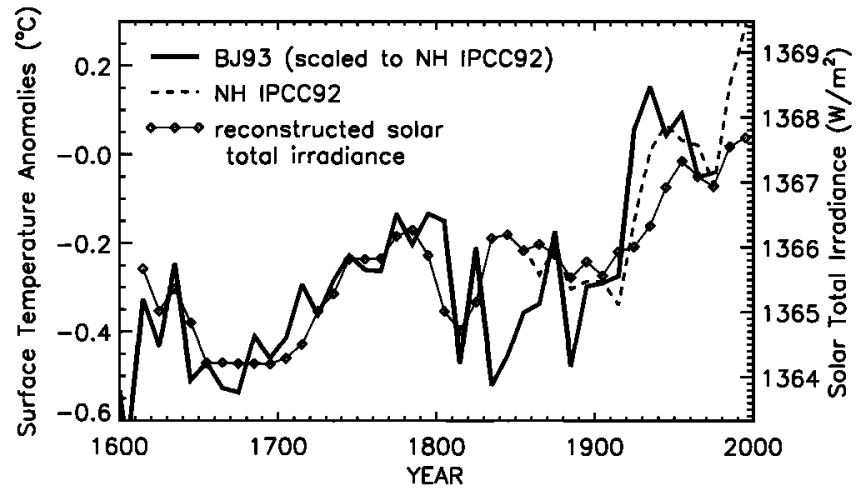

Figure 4. Compared are decadally averaged values of reconstructed solar total irradiance (diamonds) and $\mathrm{NH}$ temperature anomalies from 1610 to the present. The Bradley and Jones [1993] (BJ93) NH summer temperature anomalies (solid line) have been scaled to match the IPCC [1992] NH annual data (dashed line) during the overlap period. 
of solar irradiance explain entirely the high $\mathrm{NH}$ temperatures from 1930 to 1950 , in contrast to irradiance reconstructions in which longer term variability tracks the cycle length, rather than amplitude [Friis-Christensen and Lassen, 1991; Hoyt and Schatten, 1993].

\section{Discussion}

A new reconstruction of annual solar irradiance accounts for $74 \%$ of the variance in NH surface temperature anomalies from 1610 to 1800 and $56 \%$ of the variance from 1800 to the present. Our results indicate that solar variability may have contributed a NH warming of $0.51^{\circ} \mathrm{C}$ from the seventeenth century to the present, in good agreement with a general circulation climate model simulation. About half of the observed $0.55^{\circ} \mathrm{C}$ warming from 1860 to the present may reflect natural variability arising from solar radiative forcing, although since 1970 less than one third of the $0.36^{\circ} \mathrm{C}$ surface warming is attributable to solar variability. We conclude that solar variability may have played a larger role in recent global temperature change than has hitherto been acknowledged.

While our irradiance reconstruction, which is based on parameterizations of present day solar and stellar monitoring, includes a relatively well constrained Schwabe cycle component, the longer term variability component is by necessity more speculative. Good agreement between the reconstructed irradiances and the cosmogenic solar variability archives gives credibility to our approach, but differences with another irradiance reconstruction based on Schwabe cycle length (rather than amplitude) underscore the incomplete understanding of solar variability derived from a mere 15 years of monitoring.

Given the apparent climate system response to solar irradiance variability implied by our results, continued space-based solar irradiance monitoring is important to quantify natural solar forcing of future climate. Also needed are improved understanding of the physical causes of mutual irradiance and cosmogenic isotope variability, and of the pathways by which the climate system responds to direct as well as indirect solar radiative forcing, such as potentially associated with UV irradiance variability demonstrated by our historical UV irradiance reconstruction.

Acknowledgments. Data were kindly provided by D. Hoyt, $R$. Willson, M. Stuiver, R. Healy and the DOE/CDIAC. Discussions with D. Rind are appreciated. Helium $1083 \mathrm{~nm}$ EW data are produced cooperatively by NSF, NASA and NOAA. The DoD/SERDP provided partial support for J.L. R.B. thanks NSF and DoE for support.

\section{References}

Allen, M.R., and L.A. Smith, Investigating the origins and significance of low-frequency modes of climate variability, Geophys. Res. Lett., 21, 883-886, 1994.

Baliunas, S., and R. Jastrow, Evidence for long-term brightness changes of solar-like stars, Nature, 348, 520-523, 1990.

Beer, J., S.T. Baumgartner, B. Dittrich-Hannen, J. Hauenstein, P. Kubik, C. Lukasczyk, W. Mende, R. Stellmacher, and M. Suter, Solar variability traced by cosmogenic isotopes, in The Sun as a Variable Star, edited by J.M. Pap, C. Fröhlich, H.S. Hudson, and S.K. Solanki, Cambridge University Press, 291-300, 1994.

Bradley, R.S., and P.D. Jones, "Little Ice Age" summer temperature variations: their nature and relevence to recent global warming trends, The Holocene, 3(4), 367-376, 1993.
Eddy, J.A., The Maunder Minimum, Science, 192, 1189-1202, 1976.

Foukal, P., and J. Lean, An empirical model of total solar irradiance variation between 1874 and 1988, Science, 247, 556-558, 1990.

Friis-Christensen, E., and K. Lassen, Length of the solar cycle: An indicator of solar activity closely associated with climate, Science, 254, 698-700, 1991.

Haigh, J.D., The role of stratospheric ozone in modulating the solar radiative forcing of climate, Nature, 370, 544-546, 1994.

Hoyt, D.V., and K.H. Schatten, A discussion of plausible solar irradiance variations, 1700-1992, J. Geophys. Res., 98, 18,89518,906, 1993.

Hoyt, D.V., K.H. Schatten, and E. Nesmes-Ribes, The one hundredth year of Rudolf Wolf's death: Do we have the correct reconstruction of solar activity?, Geophys. Res. Lett., 21, 20672070, 1994.

Intergovernmental Panel on Climate Change, Climate Change 1992, Supplement to the IPCC Scientific Assessment, edited by J.T. Houghton, B.A. Callander, and S.K. Varney, Cambridge University Press, 1992.

Kelly, P.M., and T.M.L. Wigley, Solar cycle length, greenhouse forcing and global climate, Nature, 360, 328-330, 1992.

Lean, J., Contribution of ultraviolet irradiance variations to changes in the Sun's total irradiance, Science, 244, 197-200, 1989.

Lean, J., A. Skumanich, and O. White, Estimating the Sun's radiative output during the Maunder Minimum, Geophys. Res. Lett., 19, 1591-1594, 1992.

Lean, J., O.R. White, and A. Skumanich, On the solar ultraviolet spectral irradiance during the Maunder Minimum, Global Biogeochemical Cycles, 9, 171-182, 1995.

Lee III, R.B., M. A. Gibson, R.S. Wilson, and S. Thomas, Longterm total solar irradiance variability during sunspot cycle 22 , J. Geophys. Res., 100, 1667-1675, 1995.

Mann, M.E., and J. Park, Global-scale modes of surface temperature variability on interannual to century timescales, $J$. Geophys. Res., 99, 25,819-25,833, 1994.

Parker, D.E., P.D. Jones, C.K. Folland, and A. Bevan, Interdecadal changes of surface temperature since the late nineteenth century, J. Geophys. Res., 99, 14,373-14,399, 1994.

Reid, G., Solar total irradiance variations and the global sea surface temperature record, J. Geophys. Res., 96, 2835-2844, 1991.

Rind, D., and N.K. Balachandran, Modeling the effects of UV variability and the $\mathrm{QBO}$ on the troposphere/stratosphere system. Part II: The troposphere, J. Climate, 8, 2080-2095, 1995.

Rind, D., and J. Overpeck, Hypothesized causes of decade-tocentury climate variability: Climate model results, Quat. Sci. Rev., 12, 357-374, 1993.

Schlesinger, M.E., and N. Ramankutty, Implications for global warming of intercycle solar irradiance variations, Nature, 360 , 330-333, 1992.

Stuiver, M., and T.F. Braziunas, Sun, ocean, climate and atmosphere ${ }^{14} \mathrm{CO}_{2}$ : an evaluation of causal and spectral relationships. The Holocene, 3(4), 289-305, 1993.

White, O.R., A. Skumanich, J. Lean, W.C. Livingston, and S.L. Keil, The Sun in a noncycling state, Publ. Astron. Soc. Pac., 104, 1139-1143, 1992.

Willson, R.C., and H.S. Hudson, A solar cycle of measured and modeled total irradiance, Nature, 351, 42-44, 1991.

J. Lean, Code 7673L, Naval Research Laboratory, Washington, DC 20375 (email: lean@demeter.nrl.navy.mil)

J. Beer, EAWAG, CH-8600 Dübendorf, Switzerland (email: beer@hermes.eawag.ch)

R. Bradley, Department of Geosciences, Univ. of Massachusetts, Amherst, MA 01003 (email: rbradley@climate1.geo.umass.edu)

(Received June 15, 1995; revised August 18, 1995; accepted August 28, 1995.) 